\title{
Procesos de la gestión de inventario en los concesionarios del sector automotriz del municipio Cabimas
}

\author{
Inventory management processes in dealers in the automotive sector of the \\ municipality Cabimas
}

María Inciarte

gabyinciarte@gmail.com ORCID: 0000-0002-0184-5596

Publicado en enero 2020
Universidad del Zulia - Venezuela
Palabras clave

ABSTRACT

Keywords
La investigación tuvo como propósito analizar el proceso de la gestión de inventario en los concesionarios del sector automotriz del municipio Cabimas. La metodología fue de tipo analítica con un diseño no experimental, transeccional y de campo. La población estuvo constituida por 6 sujetos de los concesionarios del municipio Cabimas. Como técnica se empleó la encuesta utilizando como instrumento un cuestionario, de (21) afirmaciones con cinco (5) alternativas de respuesta: siempre, casi siempre, a veces, casi nunca y nunca. La validación se realizó a través del juicio de 5 expertos y la confiabilidad con el coeficiente de Alpha Cronbach arrojando un resultado de muy alta confiabilidad de 0.94. Los resultados arrojaron que el proceso de la gestión de inventario en los concesionarios del sector automotriz del municipio Cabimas es alta presencia, con una muy alta presencia en el pedido (4.52) y disponibilidad (4.12) y alta presencia en el almacenamiento (3.87).

Gestión de inventario, proceso de la gestión de inventario, pedido, disponibilidad y almacenamiento

The purpose of the investigation was to analyze the inventory management process in the controls of the automotive sector of the Cabimas municipality. The analytical method was of a non-experimental, transectional and field design. The population consisted of 6 subjects from the subjects of the Cabimas municipality. As a technique, the survey was used using a questionnaire (21) statements with five (5) alternative answers as an instrument: always, almost always, sometimes, almost never and never. Validation was carried out through the judgment of 5 experts and reliability with the Alpha Cronbach coefficient, yielding a very high reliability result of 0.94 . The results showed that the inventory management process in the servers of the automotive sector of the Cabimas municipality is high presence, with a very high presence in order (4.52) and availability (4.12) and high presence in storage (3.87).

Inventory management, inventory management process, order, availability and storage 


\section{INTRODUCCIÓN}

En el contexto actual, las organizaciones emprendedoras centran sus esfuerzos en el mejoramiento de los procesos operativos, siendo estos funcionales y estratégicos. En este sentido, una base importante de la gerencia se centraliza en la planificación y ejecución de tareas que permitan mejorar ejes relevantes en la empresa, como es la cadena de suministros para un determinado producto o servicio por medio del cual se busca la optimización del proceso, haciendo de esta estrategia un papel fundamental en una corporación.

La necesidad de fortalecer los procesos organizacionales se convierte en prioritaria, donde el proceso de gestión de inventarios, pudiera permitir a la empresa una planificación sobre la base de la comercialización de sus bienes, específicamente en su cadena de abastecimiento, además de disponer de los recursos con los que cuenta para alcanzar los objetivos establecidos, garantizando de esa forma la continuidad de las operaciones y la confiabilidad del proceso productivo.

En esta perspectiva, a juicio de Pulido (2009), la gestión de inventarios se define como todo lo relativo al control, manejo de las existencias de determinados bienes, en la cual se aplican métodos como estrategias que pueden hacer rentable y productivo la tendencia de estos bienes, sirviendo a la vez para evaluar los procedimientos de entradas y salidas de dichos productos.

Según los autores, Casanovas y Cuatrecasas (2010), consideran relevante que el objetivo de la gestión de inventarios es la de proveer los productos o servicios exigidos por el cliente para lo cual se necesita contar con un nivel de existencia y cantidad óptima para llevar a cabo un proceso de producción, donde ninguna organización puede iniciar sus operaciones logísticas hasta que no exista el pedido correspondiente y se concrete la operación a la clase, cantidad y momento exigido por la demanda.

Por otro lado, Méndez (2014) define el inventario como el conjunto diverso de materiales o productos que son almacenados por las empresas para ser vendidos o utilizados como materia prima en los procesos productivos llevados a cabo o los servicios prestados por la organización. Se añade la importancia vital del mismo, principalmente para los fabricantes, además este debe estar en un lugar adecuado que permita su conservación en buenas condiciones. Por otro lado, para Render (2005) la gestión de inventarios implica la existencia de todos los recursos utilizados dentro de la organización para desarrollar sus actividades y darle continuidad a cada uno de los procesos.

En ese mismo orden de ideas, para los autores previamente citados uno de los problemas más trascendentes en la gestión de inventarios es la existencia de excesos y faltantes, como consecuencia de tener demasiado de lo que no se vende $o$ se consume o muchos agotado de lo más vendido o consumido. Ello permite deducir que una verdadera gestión de inventario se centra en el equilibrio de aplicación, en diversificar los procesos en un sistema de control de entrada y salida de los productos manejados en el inventario a través de la utilización de estrategias, $\mathrm{O}$, como se realiza actualmente, con la ayuda de paquetes computacionales de simulación que mejoran el proceso antes descrito. 
Para la investigadora, la gestión de inventario se refiere a la administración fluida de productos - bien sea un servicio a prestar de cada organización, tomando en cuenta la demanda, costes de inventario, tiempo de suministro; entre otros, para así poder direccionar con eficacia las operaciones de las empresas reduciendo así al mínimo los costes de producción y a su vez los costes por perdidas.

En este sentido, para Martínez (2007), el proceso de la gestión de inventarios es la eficiencia en el manejo adecuado a través del registro, de la rotación y evaluación del inventario de acuerdo a como se clasifique y qué tipo de inventario tenga la empresa, ya que a través de todo esto se determinaran los resultados (utilidades o pérdidas) de una manera razonable, pudiendo establecer la situación financiera de la empresa y las medidas necesarias para mejorar o mantener dicha situación.

De igual forma, Cooper (2005) manifiesta que este proceso contempla diversos aspectos de la responsabilidad sobre los inventarios y afectan a muchos departamentos, y cada uno de éstos ejerce cierto grado de control sobre los productos a medida que los mismos se mueven a través de los distintos procesos de inventarios. Todos estos controles que abarcan, desde el procedimiento para desarrollar presupuestos y pronósticos de ventas y producción hasta la operación de un sistema de costo en el departamento de contabilidad para la determinación de costos de los inventarios, constituye el sistema del control interno de los inventarios, las funciones generales son: pedidos, disponibilidad y almacenamiento.

En este orden, la investigadora expresa que a las empresas no les queda otra vía que competir, asumiendo algunas de las acciones antes señaladas como una necesidad fundamental y prioritaria, requiriendo procesos gerenciales, administrativos y operativos más eficientes, influyentes de manera definitiva en los resultados alcanzados por las organizaciones, las cuales tendrán que enfrentarse e impulsar el cambio hacia nuevas formas de desarrollar sus productos o prestar sus servicios.

Es por ello, que Díaz (2006), expresa que para una correcta gestión de pedido implica:

- Conocer los requerimientos del cliente de manera precisa en lo que respecta al producto, la cantidad, los plazos, la entrega y el pedido.

- Asegurar plazos y fechas de entrega precisas, es decir, confirmar las fechas de entregas.

- Definir los parámetros de cantidad, por ejemplo los tamaños mínimos de empaque y otros aspectos relacionados.

- Mantener informado al cliente a lo largo de la vida del pedido.

- Trasferir eficientemente la información de los pedidos a la cadena de suministros.

Para Lambert y Cooper (2000), define pedido como un compromiso en firme de dos partes (proveedor y cliente) que reúne todas las condiciones mínimas necesarias para establecer una relación comercial entre ellas de manera que una de las partes (el proveedor) pone a disposición de la otra (cliente) los productos o servicio comprendidos bajos las condiciones pactadas.

Según la investigadora, es una petición u orden formal para compras con el fin de obtener 
una cantidad determinada de material o un servicio requerido especificando; característica, calidad, precio, fechas de solicitud y entrega. El compromiso de ambas parte involucradas (proveedor/cliente) garantizan los lazos comerciales.

En el contexto Venezolano, existen aspectos importantes en materia de gestión de inventario que mejorar, uno de ellos es el cambio de estructura para insertarse en el mercado mundial. Dicho cambio de estructura viene causado por dos agentes principales: el primero es la globalización de los mercados que produce una descentralización económica, y el segundo es la transición de los mercados de oferta a los de demanda, pasando de la producción en masa a la personalización en masa. Puntualizaron aspectos a mejorar en las empresas venezolanas, como: procesos inadecuados en la gestión de inventario, disposición física de los almacenes, disponibilidad y fiabilidad de la información debido a la introducción manual de datos.

De este contexto no escapan las empresas del sector automotriz del Municipio Cabimas (Jeep, Ford, Kia Motors y Chevrolet), lo cual se pudo evidenciar a través de entrevistas no estructuradas realizadas a empleados de los concesionarios, arrojando estas que existe una entrada y salida de repuestos; estos deben llevar un control de la mercancía vendida para sistematizar la disponibilidad y la posibilidad de venta ante los clientes, y a su vez elaborar pedidos con respecto a lo necesitado para así cumplir con la demanda de clientes directos.

En atención a lo expuesto, la problemática se evidencia el déficit que impide el óptimo desenvolvimiento del proceso de la gestión de inventarios en el contexto indicado, y se hace mención con la poca existencia de partes y repuestos en relación con los proveedores; ya que dichos concesionarios cuentan con sistemas automatizados para el control de inventario y los mismos no son bien utilizados, porque los procesos se registran de forma manual, además, de que estos al hacer pedidos a sus proveedores no toman en cuenta la demanda de sus productos en el mercado, y como consecuencia en muchas ocasiones quedan lotes de productos estancados en el almacén y aunque esto sea en pequeñas cantidades, este exceso de productos trae graves daños económicos.

En consecuencia, la finalidad de esta investigación se centra en el análisis del proceso de la gestión de inventario del sector automotriz del municipio Cabimas con el propósito de ver cómo se pueden mejorar los procesos de inventarios de partes y repuestos disponibles en los concesionarios, garantizando la satisfacción del cliente y por ende maximizando los beneficios.

\section{MATERIALES Y METODO}

Esta investigación por la profundidad del problema y el objetivo planteado fue analítica, con un diseño no experimental, transeccional y de campo. En este sentido, la población considerada estuvo conformada por las personas que se desempeñan en el área de inventario de los tres (3) concesionarios del municipio Cabimas, ocupando posiciones gerenciales, como se muestra en el tabla 1. 
Tabla 1. Distribución de la población

\begin{tabular}{cccc}
\hline Concesionario & Gerente General & Gerente de Repuestos & Total \\
\hline Jeep/Dodge & 1 & 1 & 2 \\
Kya Motors & 1 & 1 & 2 \\
Chevrolet & 1 & 1 & 2 \\
Total & $\mathbf{3}$ & $\mathbf{3}$ & $\mathbf{6}$ \\
\hline
\end{tabular}

Fuente: Elaboración propia (2020)

En el caso particular de este estudio se aplicó la técnica de la encuesta, con un instrumento tipo cuestionario, dirigido a los concesionarios del sector automotriz del municipio Cabimas. El cuestionario estuvo constituido por veintiuna (21) afirmaciones con cinco (5) alternativas de respuestas: siempre, casi siempre, a veces, casi nunca y nunca.

Para la validación del instrumento se aplicó la técnica de "Juicio de expertos" sometiendo a la consideración de evaluadores profesionales, quienes por su experiencia efectuaron la correspondiente revisión, siendo para esto cinco (5) profesionales. En cuanto a su confiabilidad se empleó el método de coeficiente Alpha de Cronbach alcanzando una muy alta confiabilidad del instrumento de 0,94 al incluir todos los ítems. Utilizando los aportes de la estadística descriptiva por medio de la media, se analizaron e interpretaron los datos recolectados. Luego se establecieron parámetros de interpretación de los resultados alcanzados del evento de estudio, con de diseño del baremo que se presenta en el tabla 2.

Tabla 2. Baremo para la interpretación de la media o promedio

\begin{tabular}{ccc}
\hline Alternativa & Rango & Categoría \\
\hline Siempre & $4.21-5$ & Muy alta presencia \\
Casi siempre & $3.41-4.20$ & Alta presencia \\
A veces & $2.61-3.40$ & Moderada presencia \\
Casi nunca & $1.81-2.60$ & Baja presencia \\
Nunca & $1-1.80$ & Muy baja presencia \\
\hline
\end{tabular}

Fuente: Elaboración propia (2020)

RESULTADOS Y DISCUSION

En la tabla 3 se presentan en resumen los resultados arrojaron de la dimensión pedido con una categoría de muy alta presencia correspondiente a la generación de orden de compra (4.83), dado que siempre en los concesionarios existen formatos de orden de pedido, y utilizan el duplicado para constatar la operación. Asimismo, el seguimiento de los pedidos (4.78), se observó que siempre se verifican los pedidos recibidos en el almacén, las empresas supervisan los pedidos entregados a 
los clientes, y se garantiza la entrega en el tiempo acordado con el cliente. Por otra parte, la recepción de los pedidos también alcanzo una muy alta presencia (4.67); ya que, los encuestados opinaron siempre existe una relación proveedor/cliente para cumplir los objetivos, conocen los lineamientos a seguir al momento de recibir los pedidos. De igual forma, los tipos de pedido (4.25) reflejaron una muy alta presencia, debido a que siempre se poseen controles para clasificar los pedidos, y a las condiciones de operación.

En cuanto, al indicador tiempo de entrega presento una categoría de alta presencia (4.06), ya que, los encuestados opinaron casi siempre los concesionarios poseen existencia de inventario que garantice el despacho, y el cliente recibe el pedido en el periodo establecido.

Tabla 3. Pedido

\begin{tabular}{lcl}
\hline \multicolumn{1}{c}{ Indicadores } & Promedio & Categoría \\
\hline Generación de orden de compra & 4,83 & Muy alta presencia \\
Seguimiento de los pedidos & 4,78 & Muy alta presencia \\
Recepción de los pedidos & 4,67 & Muy alta presencia \\
Tipos de pedido & 4.25 & Muy alta presencia \\
Tiempo de entrega & 4,06 & Alta presencia \\
Dimensión & $\mathbf{4 , 5 2}$ & Muy alta presencia \\
\hline
\end{tabular}

Fuente: Elaboración propia (2020)

Para la dimensión disponibilidad que se mostró en la tabla 4, los resultados arrojaron una categoría de muy alta presencia correspondiente a la confiabilidad de la entrega (4.25), ya que la población opino siempre en los concesionarios se poseen mecanismos que mantengan actualizada la base de datos, y se hacen las entregas a tiempo según lo planificado. Por otro lado, la alta presencia (4.17) correspondiente al precio, obedece a que los encuestados opinaron que siempre en los concesionarios se aplica un estudio de marketing para comparar con la competencia el valor de la mercancía, y casi siempre varían el valor de la mercancía de acuerdo a las características del pedido.

Tabla 4. Disponibilidad

\begin{tabular}{lcc}
\hline \multicolumn{1}{c}{ Indicadores } & Promedio & Categoría \\
\hline Precio & 4,17 & Alta presencia \\
Confiabilidad de la entrega & 4,25 & Muy alta presencia \\
Dimensión & $\mathbf{4 , 2 1}$ & Muy alta presencia \\
\hline
\end{tabular}

Fuente: Elaboración propia (2020) 
Visto así, para la dimensión disponibilidad se demostró un promedio de 4.21 reflejando una muy alta presencia siendo congruente con lo planteado por Amaya (2011), significa la probabilidad de que un pedido pueda ser servido íntegramente a partir del stock comercial disponible para la venta.

Para la dimensión almacenamiento que se mostró en la tabla 5, los resultados arrojaron una categoría de alta presencia correspondiente al espacio de almacenamiento propio (4.17), ya que opinaron que casi siempre en los concesionarios se disponen de flexibilidad en el diseño de los almacenes, y cuentan con el espacio suficiente para almacenar la mercancía. Ahora bien, los modelos de almacenamiento igualmente presentan una alta presencia (3.56), por cuanto casi siempre los concesionarios utilizan equipos de tecnología para el almacenamiento, y se cuenta con medio de almacenajes móviles y fijos.

Tabla 5. Almacenamiento

\begin{tabular}{lcl}
\hline \multicolumn{1}{c}{ Indicadores } & Promedio & Categoría \\
\hline Espacio de almacenamiento propio & 4,17 & Alta presencia \\
Modelos de almacenamiento & 3,56 & Alta presencia \\
Dimensión & $\mathbf{3 , 8 7}$ & Alta presencia \\
\hline
\end{tabular}

Fuente: Elaboración propia (2020)

Es así como, el almacenamiento alcanzo un promedio de 3.87 reflejando una alta presencia corroborando que los concesionarios del sector automotriz del municipio Cabimas casi siempre cumplen los criterios de almacenamiento en concordancia con lo expuesto por Schroeder (2007), el almacén es el recinto donde se realizan las funciones de recepción, manipulación, conservación, protección y posterior expedición de producto. Los principales objetivos son los de minimizar la manipulación de los productos y las operaciones de movimiento y deposito, así como maximizar la flexibilidad de las operaciones.

En la tabla 6, se reflejan los resultados del proceso de la gestión de inventario con un promedio igual a 4.20, con una categoría de alta presencia; pedido (4.52) y disponibilidad (4.21) con una muy alta presencia y almacenamiento (3.87) con una alta presencia, lo cual permite indicar que los procesos analizados están presentes en los concesionarios del sector automotriz del municipio Cabimas.

Tabla 6. Proceso de la gestión de inventario

\begin{tabular}{lcc}
\hline \multicolumn{1}{c}{ Dimensión } & Promedio & Categoría \\
\hline Pedido & 4,52 & Muy alta presencia \\
Disponibilidad & 4,21 & Muy alta presencia \\
Almacenamiento & 3,87 & Alta presencia \\
Variable & 4,20 & Alta presencia \\
\hline
\end{tabular}

Fuente: Elaboración propia (2020) 
En este sentido, los hallazgos encontrados coinciden altamente con lo citado por Martínez (2007), para quien el proceso de gestión de inventarios es la eficiencia en el manejo adecuado a través del registro, de la rotación y evaluación del inventario de acuerdo a como se clasifique y que tipo inventario tenga los concesionarios, ya que a través de todo determinan sus beneficios de una manera razonable, estableciendo su situación financiera y las medidas imperiosas para optimizar dicha situación.

\section{CONCLUSIONES}

En cuanto a los hallazgos expuestos que permitieron analizar los procesos de la gestión de inventario en los concesionarios del sector automotriz del municipio Cabimas, se pudo evidenciar que existe una alta presencia de los procesos en la gestión de inventario que llevan a cabo estos concesionarios, posicionándose en una muy alta presencia la dimensión pedido, que según los resultados obtenidos de acuerdo a la muy alta presencia de los indicadores generación de orden de compra, seguimiento y recepción de pedidos, tipo de pedido, a su vez la alta presencia en el tiempo de entrega

Asimismo, la dimensión disponibilidad reflejo una muy alta presencia en los concesionarios analizados, donde la confiabilidad de la entrega es vista por los encuestados con una muy alta presencia y el precio con alta presencia en los productos ofrecidos. No obstante aun cuando se obtuvo una categoría de alta presencia en el indicador almacenamiento y en sus indicadores estudiados como lo son espacios de almacenamiento propios y modelos de almacenamiento

\section{REFERENCIAS BIBLIOGRAFICAS}

Amaya, J. (2011). Logística Integral. Segunda Edición. Editorial ESIC. Madrid, España

Pearson. Prentice Hall. México.

Casanovas, A. y Cuatrecasas, LI. (2010). Logística empresarial. Sexta Edición. Ediciones Gestión 2000 S.A. Barcelona. España

Cooper, H. (2005) Manual Pilot (Documento en línea) Disponible: www.logistpilot.com (Consulta 2015).

Díaz, A. (2006). Gestión de inventarios en mantenimientos. Tercera edición. Ediciones ISEA. Caracas Venezuela

Glush, M. (2008). Gestión de Inventarios (Documento en línea) Disponible: http://www.cegisu_tica,cifiles/revistabeneficio s_centro_distribución.pdf, (Consulta 2015)

Lambert, D.M. y Cooper, M.C. (2000). Issues in Supply Chain Management. Industrial Marketing Management, Vol. 29

Martínez, E. (2007). Negociación y estrategias de aprovisionamiento. Cuarta edición. Fundación confemental. Madrid España.

Méndez, I. (2014). Gestión de Inventario en empresas de servicio automotriz del municipio Cabimas. Universidad del Zulia. Nucleo Costa Oriental del Lago. Coordinación de Postgrado e Investigación

Pulido, J. (2009). Gestión de Inventario. El secreto de la rentabilidad. Editorial Torino. Caracas

Render, B. (2005). Principios de Administración de Operaciones. Quinta Edición. Editorial Pearson, México

Schroeder, R. (2007). Administración de Operaciones. 2da. Edición. Editorial McGraw Hill. México 